\title{
Gradhiva
}

\section{Pièges à voir, Pièges à penser}

Présences cachées dans l'image

\section{Carlo Severi}

\section{OpenEdition}

\section{Journals}

Édition électronique

URL : http://journals.openedition.org/gradhiva/2019

DOI : 10.4000/gradhiva.2019

ISSN : 1760-849X

Éditeur

Musée du quai Branly Jacques Chirac

\section{Édition imprimée}

Date de publication : 18 mai 2011

Pagination : 4-7

ISBN : 978-2-35744-042-5

ISSN : 0764-8928

Référence électronique

Carlo Severi, «Pièges à voir, Pièges à penser », Gradhiva [En ligne], 13 | 2011, mis en ligne le 18 mai 2011, consulté le 21 septembre 2020. URL : http://journals.openedition.org/gradhiva/2019; DOI: https://doi.org/10.4000/gradhiva.2019 


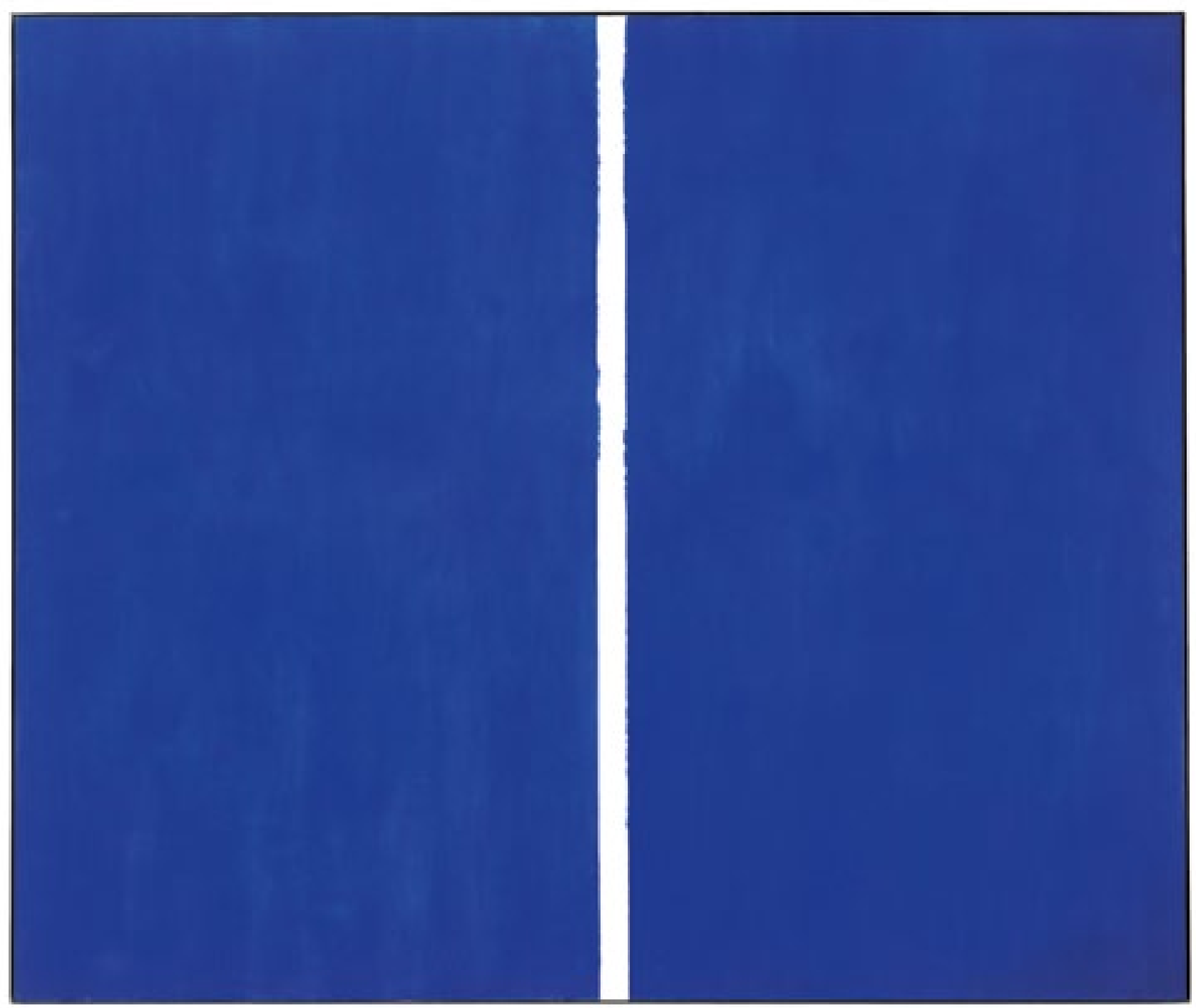

Fig. 1 Barnett Newman, Onement VI, 1953, huile sur toile $(259 \times 305 \mathrm{~cm})$. Collection particulière $\odot$ ADAGP, Paris 2011/Photo Courtesy The Barnett Newman Foundation. 


\section{Pièges à \\ Pièges à voir \\ penser \\ Présences cachées dans l'image}

Carlo Severi

-

1. Paris-Berlin, 12 juillet

-6 novembre 1978,

Centre Georges-Pompidou, Paris.

2. Magiciens de la terre, 18 mai-28 août 1989 , Centre Georges-Pompidou. Catalogue éponyme publié par les éditions du Centre GeorgesPompidou, Paris, 1989.

\section{Présentation}

Ce dossier est consacré à une réflexion, entre anthropologues et historiens de l'art, sur le rôle de l'ambiguïté dans la représentation visuelle. Potentielle, double ou chimérique, l'image ambiguë a récemment suscité la réflexion, dans les deux disciplines, en termes nouveaux. Pour en rendre compte, nous avons réuni des recherches menées par des ethnologues et des réflexions suscitées par une grande exposition, Une image peut en cacher une autre, où le thème de l'ambiguïté de l'image était appréhendé du point de vue de l'histoire de l'art. Dans un entretien avec Carlo Severi et Julien Bonhomme, Jean-Hubert Martin situe l'enjeu de cette exposition dans le cadre du travail, qu'il mène depuis de longues années, pour ouvrir aux arts non occidentaux le cercle fermé du monde de l'art. En 1978, avec Werner Spies, Martin dirigeait l'exposition Paris-Berlin ${ }^{1}$ au Centre Pompidou. Certains historiens de l'art auraient pu s'inspirer du primitivisme des expressionnistes pour passer à l'étude des arts non occidentaux. D'autres, comme Rubin, ont fait référence à l'œuvre de Picasso. Plus radical, Martin s'est inspiré de Dada : «Dans Dada - écrit-il -, il y avait déjà, d'une part, l'idée de prôner l'égalité des œuvres et d'autre part, celle de se battre contre le culte héroïque de l'artiste. Le programme des Magiciens de la terre était là.» C'est à partir de ce projet qu'une série d'expositions est née (de Partage d'exotismes à Altären, à Artempo), où il est possible d'aller au-delà des conceptions traditionnelles de l'espace et du temps de l'art occidental, pour mobiliser d'autres jeux de regard, et d'autres formes d'exercices de la pensée visuelle. 
-

3. Mach 1886 : 83. Voir l'article de Dario Gamboni.

4. Souriau 1909 [1893] : 8 ?.

5. Nous tenons à témoigner de notre reconnaissance pour le Capes et le Cofecub, qui ont permis à ces recherches de se réaliser dans le cadre d'un projet de coopération franco-brésilienne («Art-image-mémoire »), placé sous la responsabilité de Carlos Fausto (Museu Nacional, Rio de Janeiro) et Carlo Severi (EHESS-CNRS, Paris).
Dario Gamboni, pour sa part, reconstruit une fascinante histoire des nuages en tant que forme privilégiée de l'ambiguïté visuelle (et du type de projection qu'elle suscite), qui traverse l'histoire de l'art européen de la Renaissance à la modernité. Depuis qu'il y a des nuages et des êtres humains - écrit Gamboni-les hommes y ont reconnu des images. Dès l'Antiquité, l'interprétation des nuages est conçue comme le produit de l'inclination naturelle des hommes à imiter. Le dialogue avec les nuages devient un modèle mental de l'activité artistique : l'œuvre de l'artiste, qui naît lorsque l'imitation s'effectue avec la main et non seulement avec l'esprit, ne fait que traduire ce dialogue en actes. Plus tard, lorsque la nature cesse d'être vue comme un artiste capable de s'imiter lui-même, l'étude des nuages se lie avec l'étude de la perception. Ernst Mach comptait «la projection de phantasmes sur ce qui n'est pas vu clairement " parmi les "manifestations de l'imagination et de la mémoire au sein des perceptions sensorielles ${ }^{3}$. Paul Souriau, de son côté, écrivait en 1893 que « regarder un dessin, c'est voir des chimères dans les nuages ${ }^{4}$.

En dialogue avec ces historiens de l'art, des ethnologues interrogent, à partir d'autres horizons, la notion de chimère. Tel qu'il a été défini jusqu'à présent, ce type de représentation désigne essentiellement un phénomène visuel, fondé sur la figuration et sur la relation binaire qui peut s'établir par la représentation conjointe de deux êtres différents. Les recherches ${ }^{5}$ réunies dans ce dossier de Gradhiva mettent, chacune à leur manière, cette première définition à l'épreuve. Dans l'essai qu'il consacre au "Masque de l'animiste», Carlos Fausto montre que l'on peut comprendre, dans l'espace chimérique, des représentations plus complexes, qui ne sont plus seulement engendrées à partir de l'articulation entre deux images antagonistes. Certains masques amérindiens offrent l'exemple de cette complexité imprévue. Le paradoxe du masque, écrit Carlos Fausto - est que tout le monde sait qu'à l'intérieur, ne se trouve pas un esprit, mais un membre de sa propre communauté. Cependant, un masque sans personne pour le porter demeure un simple objet inerte. Par le déguisement qu'il réalise, le masque donne en effet lieu à une attribution de subjectivité déplacée. Mais comment l'absent se rend-il présent (et le présent, absent) ? Comment le visible recule-t-il pour céder la place à un invisible rendu visible par le moyen du masque? Pour rendre compte, en termes visuels, de ce déplacement d'identité, Fausto distingue entre deux notions formelles, celle d'" emboîtement récursif " et celle de "référence multiple».

Composés de véritables séries de termes différents, qui s'inscrivent dans des niveaux d'appréhension enchâssés les uns dans les autres, les masques que Fausto analyse développent donc de manière nouvelle la logique de la représentation chimérique. Poursuivant sur ce chemin, les recherches de Tommaso Montagnani et Bruna Franchetto sur les chants kuikuro ouvrent aujourd'hui un horizon entièrement nouveau, qui implique, dans la représentation chimérique, une articulation jusque-là inédite entre le son et la parole. Dans la tradition kuikuro, l'union de la musique venant du répertoire masculin et du texte venant du répertoire de chants féminins donne lieu à la naissance d'une entité sonore qui est à la fois un humain, un esprit et un animal. Chacun des deux éléments (mélodie et texte) de cet objet musical complexe possède une existence et une fonction autonomes dans deux contextes rituels séparés : une mélodie est associée au nom d'un esprit dans le rituel masculin, et un nom propre à un personnage humain dans le rituel féminin. La combinaison de la musique instrumentale et 
d'un texte poétique crée ainsi une nouvelle dimension rituelle dans laquelle des identités humaines et surnaturelles sont superposées dans une représentation chimérique de type sonore.

De son côté, Els Lagrou montre comment plusieurs techniques formelles utilisées dans le graphisme des Indiens cashinahua et d'autres "peuples à dessin " peuvent être vues comme des techniques perspectivistes, qui aident à visualiser la potentialité de transformation des phénomènes perçus. Cette analyse, focalisée sur la notion de cadrage, permet d'introduire la notion nouvelle de chimère abstraite, que l'on se gardera, naturellement, de confondre avec celle d'aniconicité. Si on peut appeler ces dessins abstraits, c'est parce qu'ils thématisent, en tant qu'univers de la représentation, non pas l'imitation du réel, mais une expérience spécifique du regard. Carlo Severi reprend, en termes théoriques, la définition du concept de chimère de trois point de vue : morphologique, logique et esthétique. Il propose ensuite de passer de la définition d'une typologie des représentations, à l'identification d'une logique des relations représentées par l'image. Il s'agit en effet de montrer, à travers l'analyse de deux traditions iconographiques amazoniennes (des Yekwana et des Wayana), comment la désignation par projection d'une présence imputée engendre l'idée d'une essence propre aux êtres chimériques. La représentation chimérique s'associe ainsi à la définition essentialiste d'une classe d'existants dont les traits définitoires ne coïncident jamais avec ceux qui, au sein du monde humain, animal ou végétal, définissent une espèce. L'étude de la représentation chimérique permet ainsi d'éclairer d'un regard nouveau l'ontologie que ces traditions présupposent.

Un autre développement possible de la notion de chimère concerne, en Occident comme ailleurs, la notion de mouvement. Dans la convention visuelle de la perspective occidentale, la traduction de traces inscrites sur une surface plane ne se traduit pas seulement en indications de profondeur, mais aussi en indications de mouvement. En tant que témoignage d'une métamorphose, une image chimérique implique l'idée d'un mouvement implicite, qu'un certain travail du regard est appelé à reconstruire mentalement. Or, que se passe-t-il lorsque ce mouvement, comme dans la construction d'images mécaniques du corps humain, est rendu explicite? Est-ce qu'il devient possible de concevoir, en ces cas, des machines chimériques, ou des représentations que le mouvement même rendrait chimériques? L'essai que Joffrey Becker consacre au type de subjectivité et de mouvement qu'on attribue à certains robots apporte un éclairage tout à fait nouveau sur ces questions. Sous cet angle, les robots apparaissent comme les intermédiaires d'un mouvement récursif allant du corps imité à l'interprétation de cette imitation, telle qu'elle prend le corps pour modèle. À l'image des automates de l'âge classique, les robots constitueraient des formes de simulation et de dramatisation de la vie, illustrant ainsi une théorie de la personne à travers une renégociation des limites du vivant et de l'intelligence.

Définie dans ses traits morphologiques, logiques et esthétiques, la notion de chimère pourra, entre invisible et donné à voir, représentation iconique et indiciaire, fragment et cadre réflexif, se décliner différemment, se radicaliser, se démultiplier sur d'autres registres, suivre la voie de la projection visuelle ou celle de la mobilisation de l'inférence.

C'est cette complexité de l'image ambiguë, et l'espace de recherche que cette complexité ouvre en anthropologie et en histoire de l'art, dont ce dossier de Gradhiva vise à témoigner. 